\title{
EL FÚTBOL DE BARRIO COMO PROCESO EDUCATIVO NO FORMAL: UN ESTUDIO DE CASOS EN LA CIUDAD DE VALDIVIA, CHILE
}

\author{
O FUTEBOL DE BAIRRO COMO PROCESSO EDUCATIVO NÃO FORMAL: UM \\ ESTUDO DE CASOS NA CIDADE DE VALDIVIA-CHILE
}

NEIGHBORHOOD FOOTBALL AS A NON-FORMAL EDUCATIONAL PROCESS: A STUDY OF CASES IN THE CITY OF VALDIVIA - CHILE

\section{Katherine Daniela Benilde Peña Ojeda*, Nicolás Ricardo Mujica Hernández *, Sergio Alejandro Toro Arévalo *}

Palabras clave Deportes.

Aprendizaje. Grupo social. Fútbol.

\begin{abstract}
Resumen: El propósito de la investigación fue comprender los procesos de convivencia de un equipo de jugadores de fútbol de barrio, el "Club de Fútbol Palestino Los Jazmines" de la ciudad de Valdivia, en el sur de Chile. Se realizó una intervención en calidad de profesores de las series mini e infantil, en la cual se destacaron tres jugadores como informantes clave del estudio. En primer lugar, se realizó una revisión bibliográfica sobre el tema; luego, a partir de un diseño metodológico cualitativo basado en el paradigma socio-crítico, se analizaron e interpretaron los datos registrados en el diario de campo y en las entrevistas semiestructuradas aplicadas a los informantes clave, a sus padres y a la presidenta del club. Los resultados del estudio contribuyeron a la caracterización de las dinámicas de relación social y a la implementación y evaluación de una propuesta educativa no formal destinada a fortalecer la convivencia del equipo.
\end{abstract}

Resumo: 0 propósito desta pesquisa foi compreender os processos de convivência de uma equipe de jogadores de futebol de bairro, o "Club de Fútbol Palestino Los Jazmines", da cidade de Valdívia, no sul do Chile. Os investigadores realizaram uma intervenção na qualidade de professores das séries míni e infantil, na qual destacaram três jogadores como informantes-chave do estudo. Em uma primeira instância, se realizou uma revisão bibliográfica do tema, logo a partir de um desenho metodológico qualitativo baseado no paradigma sociocrítico, analisaram e interpretaram os dados registrados em diário de campo e nas entrevistas semiestruturadas aplicadas aos informantes-chave, seus pais e a presidenta do clube. Os resultados do estudo contribuíram para a caracterização das dinâmicas de relação social, assim como para a implementação e avaliação de uma proposta educativa não formal destinada a fortalecer a convivência da equipe.

Abstract: This research tries to understand the processes of coexistence in a neighborhood football team - "Club de Fútbol Palestino Los Jazmines", in the southern Chilean city of Valdivia. Researchers intervened as teachers of under-nine and juvenile football players and chose three players to be the key informants of the study. Firstly, a bibliographic review of the key themes was conducted. Then a methodological framework centered on a socio-critical paradigm was established, based on qualitative methodology, to analyze and interpret the data collected through a field diary and semi-structured interviews with the key informants. Results contributed to characterize the dynamics of social relations, and to implement and evaluate a non-formal educative proposal aimed at strengthening coexistence in the team.
* Universidad Austral de Chile. Valdivia, Chile. E-mail: katherine.uach@gmail.com

Recebido em: 04-11-2015 Aprovado em: 05-09-2016 (c) (1) (8) Licence 


\section{ANTECEDENTES GENERALES}

El fútbol constituye un deporte que abarca diversos contextos sociales, sexo, género, visión política, clase social. Santiago (2002) infiere que es un fenómeno social que engloba valores, normas y comportamientos que lo diferencian de otras prácticas, donde se construyen y confluyen aspectos que propenden una herramienta educativa. Balibrea (2010) indica que la intervención mediante el deporte en sectores sociales vulnerables favorece procesos educativos, pues los aprendizajes obtenidos tienen implicancia en el comportamiento de los niños tanto a nivel individual como colectivo, independiente a la situación de juego o entrenamiento.

De lo anterior, emergieron dos cuestionamientos referido al fútbol como enfoque central: primero, qué situaciones vinculadas a la relación social vulneran la convivencia y, cuáles, al contrario, la fomentan. Estas preguntas surgen de la discusión sobre la potencialidad educativa que tiene o no el fútbol, entendiendo que este deporte comprende una relación de convivencia social, la cual, al ser intencionada, se aproxima a un proceso educativo (MATURANA, 2000). En segundo lugar, detectar los beneficios que adquirieron los miembros de la comunidad del "Club Palestino" y los profesores en formación a partir de la convivencia sostenida. De esta manera, tales cuestionamientos se discutieron en profundidad en el análisis y conclusiones del estudio.

A partir de la intervención, se buscó comprobar si el fútbol del Club Palestino de la comunidad Los Jazmines, constituye un proceso educativo, considerando la evidencia de necesidades básicas en los niños en cuanto a habilidades sociales, despertando el interés por conocer el factor que incide en el grupo y determinar si el afecto, la confianza y la proximidad, se pueden fomentar desde la relación social de los niños con sus compañeros, con quienes les rodean o bien, con quienes los guían en cada encuentro deportivo. De esta forma, el propósito fue comprender la convivencia que establecen jugadores del Club a partir de sus relaciones sociales en la comunidad, en base a un diagnóstico de las habilidades sociales de los niños, desde su inclusión en instancias que emergen en torno a este deporte. Luego, desde los resultados, se diseñó, implementó y evaluó a nivel cualitativo, una propuesta educativa de carácter no formal basada en el fútbol como un agente educativo.

En complemento, cabe mencionar que una de las características principales del Club, es que la gran mayoría de sus miembros han residido por años en el barrio, lo cual permite identificar un sentido comunitario y familiar en dicho grupo.

\section{REFERENTE CONCEPTUAL}

El fútbol es entendido como un proceso educativo de carácter no formal, considerándose desde Freire ${ }^{1}$ citado en Ritterstein (2008) como una interacción entre el sujeto y el ambiente que le rodea desde el juego. Para el proceso educativo, el vínculo es el centro de la acción, ya que permite a la persona intercambiar aprendizajes con sus pares, conmutando comportamientos y percepciones de la realidad que los envuelve. Hernández y Tapia (2010), se concibe que el fútbol es un potenciador social desde lo comunitario, transmitiendo aprendizajes en base a los vínculos sociales que se establecen en su contexto de desarrollo.

Concibiendo el vínculo como proceso educativo, se destaca el aprendizaje desde la reciprocidad y el intercambio entre los sujetos que son partícipes de la relación; este vínculo

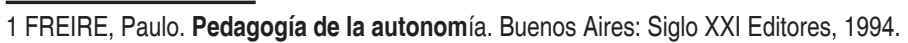


facilita el intercambio de comportamientos y acciones sociales, desprendidas desde la interacción con otros y del crecimiento mutuo. En base a esto, en Schutz (2001) sostiene que la relación es una necesidad social, con tres dimensiones: relación intrapersonal, relación interpersonal y las necesidades sociales como tales. En Ruggio (2011), se observa lo intrapersonal como la idea de que el ser humano no es un sujeto segmentado en componentes yuxtapuestos entre sí; más bien, comprende un ser integral, que se proyecta desde su complejidad a partir de una relación intrapersonal y de su auto-organización.

Así mismo, lo interpersonal según Schutz (2001) es la proyección de lo intrapersonal, entendiendo que el sujeto se encuentra inmerso en una comunidad en base a la acción de manifestar sus aprendizajes y desarrollarse como ser social; Ritterstein (2008, p.3), plantea que: "El sujeto siempre necesita de otro [...], el interjuego entre la necesidad y la satisfacción son constituyentes en el desarrollo del sujeto [...] la necesidad del otro estará siempre presente, y resulta de la experiencia y vínculo que establecemos". Por ello, las necesidades sociales son establecidas por Schutz (2001) como condiciones que forman un perfil social satisfactorio para el ser humano, complacidas en la relación social que sostiene con los demás en el contexto de desempeño. Dichas necesidades se sintetizan en tres ámbitos: la inclusión, que implica a la persona sentirse importante, significativa y acogida o excluida dentro de un grupo humano; el control, manifestado en la toma de decisiones respecto a sus pares en el contexto que participa, de acuerdo a la satisfacción de sus necesidades intrapersonales; y el afecto, que implica la proximidad de la persona con sus semejantes, en base a la concreción de sus necesidades de inclusión y control.

Las relaciones sociales que desenvuelven los sujetos participantes del fútbol, comprenden un proceso educativo que implica aprendizajes como ser único y en relación al contexto comunitario. Es un proceso, fundamentalmente, de apropiación mediante la experiencia de transformaciones significativas y tangibles que puedan ser observadas dentro de la práctica deportiva u otra manifestación de la motricidad (TORO, 2010), en este caso en el contexto del fútbol de barrios. El deporte permite el vínculo de los participantes a partir de las relaciones que se establecen dentro y fuera del juego, la convivencia es eje fundamental para el desarrollo de los sujetos en este ambiente. Esto último, corresponde a las habilidades sociales, entendidas desde Gil y León (1998) como la ejecución de conductas que responden a necesidades de comunicación intrapersonal, que Pacheco (2000) define como un conjunto de comportamientos verbales y no verbales mediante los cuales los niños influyen en su medio ambiente, obteniendo, suprimiendo o evitando consecuencias deseadas y no deseadas en el contexto social. Se adquieren en la relación desde lo aprendido por observación y en la convivencia y se da en su ambiente de desarrollo, provocando consecuencias tanto perjudiciales como beneficiosas a la convivencia.

En efecto, la convivencia constituye un conjunto de relaciones cotidianas manifestadas en diversos contextos, que involucra aprendizajes desde un vínculo en el que siempre intervienen otros y que permite adaptarse a los demás y a la situación (JIMÉNEZ ROMERO, 2005). Por ello, se acoge la idea de compartir una vivencia en particular, entendiendo que la relación supone la manifestación del desarrollo intrapersonal y la relación interpersonal, articulada a partir de comportamientos realizados en el contexto de interacción, basados en habilidades sociales. 


\section{PROPUESTA EDUCATIVA NO FORMAL}

La propuesta consideró el fútbol como un proceso educativo de carácter no formal, mediante actividades basadas en principios de ergonomía, modelos óptimos de conducta, convivencia, respeto, autoestima, sentimientos y valoraciones positivas, y cuidado e higiene personal. Los espacios de ejecución incluyeron la Escuela de Fútbol, Gimnasio e Infraestructuras de la Universidad Austral de Chile, partidos de Fútbol Profesional en un Estadio, y un restaurant de Valdivia, lugares que los informantes clave desconocían.

En conjunto con la Presidenta del Club, los niños y los padres diseñamos una pauta de evaluación diagnóstica con énfasis en la caracterización de las dinámicas de relación social y convivencia de los jugadores del equipo. Luego programamos actividades de encuentro social en diversos contextos, teniendo el fútbol como núcleo central, y finalmente, aplicamos la pauta de evaluación de habilidades sociales, con el fin de evidenciar los efectos de la intervención.

La evaluación fue realizada en el diario de campo, el cual contemplaba registros anecdóticos de cada actividad y a su vez esbozaba los aprendizajes obtenidos. En este sentido, valoramos la pertinencia de cada instancia y la observación de las reacciones y las manifestaciones ergonómicas registradas en las pautas de evaluación, puesto que evidenciaron la conducta de comodidad/incomodidad de los niños ante las situaciones nuevas.

\section{DISEÑO METODOLÓGICO}

\subsection{Paradigma y metodología de investigación}

Según Alvarado y García (2008), mediante el paradigma socio-crítico nos situamos en promover las transformaciones sociales, dando respuestas a los problemas específicos de la comunidad seleccionada, con la participación de sus miembros en dicho proceso. Por otra parte, el enfoque cualitativo, "[...] es un proceso interpretativo de indagación [...] que examina un problema humano o social. Quien investiga, construye una imagen compleja y holística, analiza palabras, presenta detalladas perspectivas de los informantes y conduce el estudio en una situación natural" (VASILACHIS, 2006, p. 24). Siguiendo este lineamiento metodológico, para el desarrollo y el análisis de los datos se basó en una Investigación-Acción Participativa, considerando que es un proceso en el cual los participantes: "[...] pasan de ser "objeto" de estudio a "sujeto" protagonista de la investigación, controlando e interactuando a lo largo del proceso [...] y necesitando una implicación y convivencia del investigador externo en la comunidad a estudiar". (ALBERICH, 2007, p. 6).

\subsection{Recolección de datos}

Se consideraron cuatro procedimientos: (1) análisis documental, que consistió en una lectura comparativa y analítica de las diferentes teorías de cada tema; (2) observación participativa, que permitió reconocer los comportamientos del grupo en cuestión, cuya información fue registrada en un diario de campo; (3) entrevistas semiestructuradas, con énfasis en profundizar en áreas no desarrolladas a cabalidad por los demás instrumentos; (4) pauta de evaluación de habilidades sociales, diseñada por el grupo de investigadores en complemento 
con lo inferido de Gutierrez (2002), Coronil (2007-2008) y Bueno y Lorenzo (2011), para valorar una de las categorías centrales de la investigación y apreciar el grado de desarrollo de los niños en cada ámbito.

Figura 1 - Diagrama metodológico.

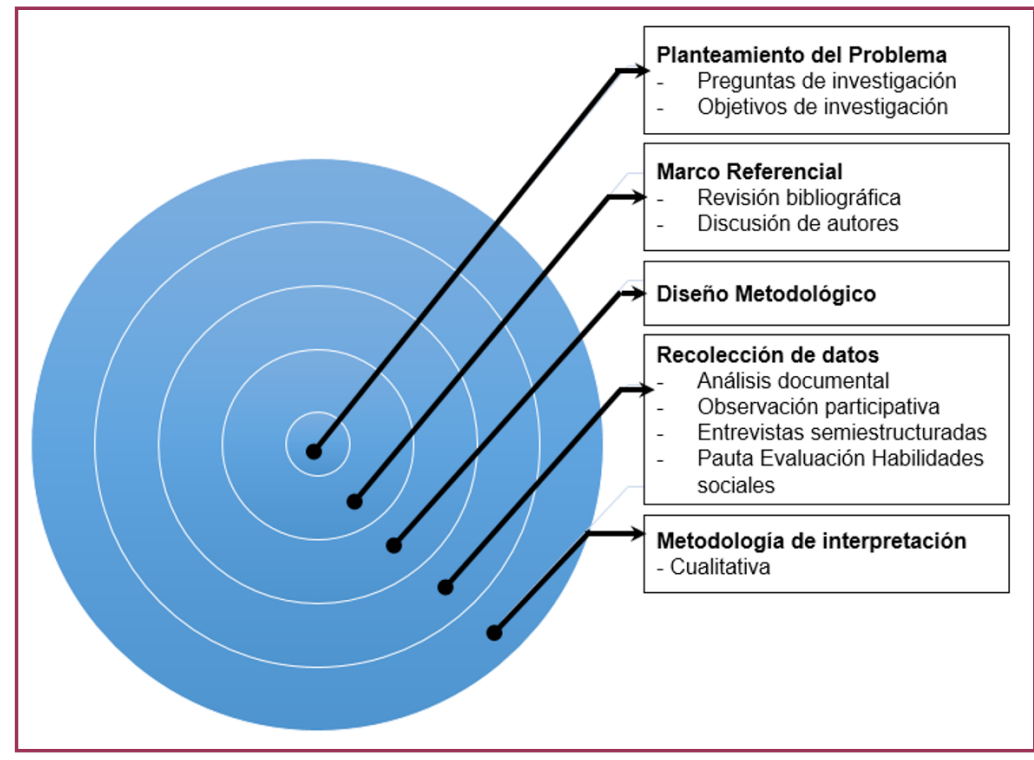

Fuente: Autores

En la Figura 1 se aprecia el flujo del proceso investigativo, donde los elementos constituyentes del estudio se relacionan entre sí, permitiendo desenvolverse dentro del paradigma de investigación socio-crítico, y de forma integrada dar curso al análisis de la investigación.

\subsection{Caracterización de informantes claves}

Los informantes claves corresponden a tres jugadores del Club; dos de ellos residen en la población "Los Jazmines" y uno alejado de la comunidad. De esta manera, la selección de los participantes se realizó en base a su participación en la Serie Infantil y porque acompañaban a los investigadores en el monitoreo de otros grupos del equipo.

En este sentido, se evidenció que la residencia o no residencia en la población Los Jazmines, es un factor que contribuye en las dinámicas de relación social que se establecen en este Club. Sin embargo, las características familiares de los informantes, por ejemplo la relación con sus padres, fueron factores que no trascendieron en las conclusiones del estudio.

\section{ANÁLISIS E INTERPRETACIÓN DE LA INFORMACIÓN}

De acuerdo a lo expresado por los informantes claves, se desprendieron los sentidos fundamentales para ser propuestos como categorías de análisis e interpretación. Posteriormente, mediante un proceso interpretativo, a contrastar y situar con el marco referencial, los objetivos de la tesis y objeto de estudio, se obtuvo como resultado las categorías en dos niveles: deductivo e inductivo, las cuales se desarrollan a continuación. 


\subsection{Nivel deductivo}

\subsubsection{Relación intrapersonal}

Se entiende como la relación que el sujeto tiene con su propia integralidad, constituyendo el sustento del vínculo con los demás. De esta forma, el análisis se basó en la interacción intrapersonal de cada integrante del grupo de informantes claves, considerando que es insatisfactorio caracterizar la relación intrapersonal de los niños, desde una postura externa a ellos.

Figura 2 - Relación intrapersonal.

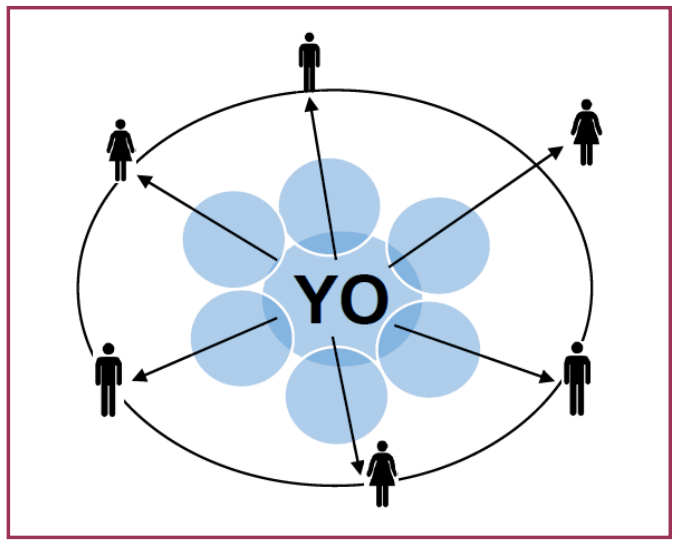

Fuente: Autores

Como se aprecia en la Figura 2, la formación intrapersonal de los niños es un proceso que se ha transformado debido a experiencias que enmarcan y determinan su relación intrapersonal. Quienes reconocen sus falencias y debilidades pueden transformarlas y orientarlas hacia un desarrollo positivo, por el contrario, en uno de los niños con experiencia negativa, se distinguió un comportamiento indiferente con los comentarios de los demás.

En el caso del Informante 3, se destaca que reconoce sus errores y permite que los demás lo apoyen, atendiendo a sus comentarios para luego retribuir a sus compañeros la ayuda que le brindan. Esto se puede argumentar a través de: "yo igual he cometido errores, y a veces me retan y a veces me apoyan. Entonces yo trato de hacer las cosas bien y apoyarlos a todos" (Fuente: Informante Clave 3). Por otro lado, en los casos de los Informantes 1 y 2, existen episodios que marcaron y delimitaron su desarrollo intrapersonal en el Club. En ambos casos, se detectaron comportamientos indiferentes hacia los comentarios tanto de sus compañeros de equipo como de las personas que están en este contexto. [...] me decían corre guatón corre... ahí sufrí en ese momento". (Fuente: Informante Clave 1) "no me interesa lo que hablen, si juego mal o juego bien" (Fuente: Informante Clave 2).

\subsubsection{Relación interpersonal}

La relación interpersonal es la visión de la persona en torno a su autoconcepción. La comprensión que el sujeto tiene de su integralidad, determina el vínculo que establece con los demás. Esto último, se evidencia a continuación: 
Figura 3 - Relación interpersonal.

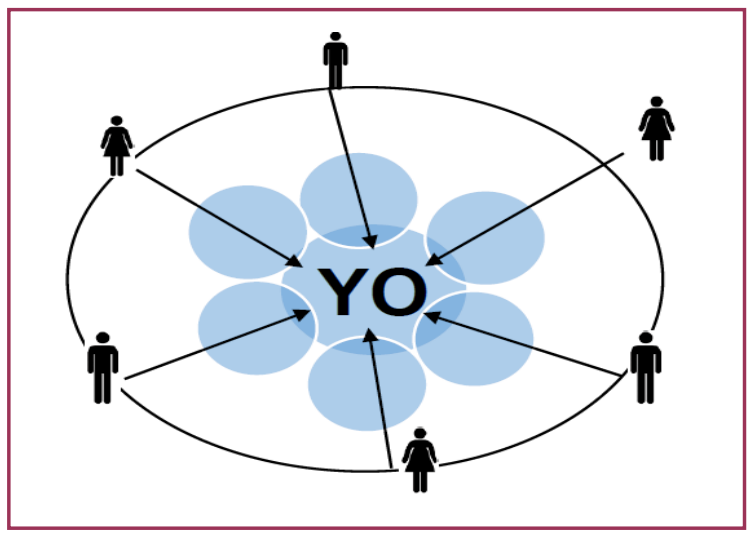

Fuente: Autores

En la figura 3 se destaca que la relación interpersonal (los otros) se construye desde la proyección que supone la relación intrapersonal (Yo). Por ello, se incorporan en esta categoría los registros de los padres de cada niño con respecto a su desarrollo, entendiendo que la imagen que proyecta hacia los demás es la que determina la convivencia dentro su contexto.

En el caso del informante clave 1, existe una conexión entre lo intrapersonal y lo interpersonal. El niño expresa un bienestar consigo mismo, que la madre puede reconocer de forma tangible: "Se acepta como es...se quiere a sí mismo... si él no se quisiera, no andaría jugando, no sería feliz [...] andaría deprimido. Y ustedes saben cómo es él, es el más alegre" (Fuente: Madre Informante Clave 1). La madre considera importante la relación intrapersonal en el vínculo interpersonal al señalar que si su hijo no estuviese conforme consigo mismo, no demostraría su felicidad jugando con sus amigos.

Por otra parte, en el informante clave 3 se distingue una contradicción entre la madre y el niño, ya que ésta señala que su hijo no se acepta tal y como es porque físicamente no está conforme. Al contrario, el niño establece que se auto-acepta y omite lo expresado por su mamá. Esto se evidencia en: "[...] tiene un problema por su porte [...] dice que es muy chico para su edad, le gustaría ser un poquito más alto [...] los niños de repente se burlan”. (Fuente: Madre Informante Clave 3). En este caso, se deduce que el niño no tuvo la confianza necesaria para transmitir su problema al grupo de investigadores, a raíz de que señala estar conforme consigo mismo, contradiciendo lo emitido por la madre, que ve en la proyección interpersonal de su hijo una preocupación importante por su estatura, produciéndole una inmensa insatisfacción; esto se evidencia en: “ ¿Te sientes bien contigo mismo? (Sí), Pero tú no compartes mucho tus cosas con tus compañeros, (No) ¿por qué? (Es que como que no tengo como la confianza como para decirles algo, lo pueden contar) (Fuente: Informante Clave 3)

Respecto a lo anterior, el informante clave 3 no convive con sus compañeros de equipo, dado que como señala anteriormente, no confía en ellos y por ende no los considera sus amigos. Esto limita la convivencia, puesto que la relación entre el niño y sus pares es únicamente a nivel deportivo. La conexión entre lo intrapersonal y lo interpersonal se ve afectada desde la proyección del niño hacia los demás. Por tanto, deducimos que omitió información considerando que el grupo de investigadores es parte del equipo y por ende, no son sus amigos.

Finalmente, se reconoce que la proyección del sujeto limita las dinámicas de relación que se sostienen en el Club Palestino, y por ende la convivencia establecida entre los jugadores. El 
recurso que permite dicha interacción, corresponde a las habilidades sociales, capacidades que permiten a las personas encontrarse a nivel social desde la manifestación de su integralidad.

\subsubsection{Habilidades sociales}

En el estudio se trabajaron diferentes habilidades sociales que permitieron comprender la convivencia que sostienen los jugadores. Los padres identificaron las habilidades sociales más frecuentes de sus hijos, y éstos últimos reconocieron desde su propio comportamiento lo siguiente: "¿te sientes muy mal si pierdes, o si ganas? (No, porque hay que saber ganar y perder), y ¿has aprendido a aceptar a diferentes personas? (sí, aprendí a aceptar) (Fuente: Informante Clave 1), "yo me preocupo harto pero a veces no puedo llegar a la hora, pero yo siempre me preocupo por ir". (Fuente: Informante Clave 2), "por ejemplo no ser insolente 0 desordenado dentro de la cancha" (Fuente: Informante Clave 3), "¿por qué crees tú que te gusta tanto ganar cada partido de fútbol, independiente de donde sea? (Es que a lo mejor me acostumbré a eso") (Fuente: Informante Clave 3).

La habilidad social más relevante en los niños es el respeto hacia sus pares, manifestada en la aceptación hacia los demás; también, la puntualidad y la responsabilidad respecto a compromisos deportivos, lo que se transmite a su vida cotidiana. La convivencia de los jugadores es influenciada por la búsqueda de satisfacer las necesidades sociales, y generar la capacidad de dar respuesta es lo que se concibe como habilidad social.

\subsection{Nivel inductivo}

\subsubsection{El fútbol de barrio en el Club Palestino Los Jazmines como proceso educativo}

El proceso educativo es inferido desde Freire citado en Ritterstein, (2008), como una praxis que comprende la interacción entre el sujeto y el medio que le rodea, implicando la relación con los otros. Por tanto, el fútbol significa un proceso educativo que permite y fomenta las relaciones entre sus participantes. Sobre esto último, las Madres de los Informantes 1 y 2 señalan lo siguiente: "Se ha puesto más activo, no está tanto tiempo metido en el computador o chatiando, pesca su pelota y se va a la cancha, o con los amigos, y lo bueno que tiene, es que comparte. (Fuente: Madre Informante Clave 1)" y "Depende donde juegue, porque le va a servir como a conocer todo tipo de personas, relacionarse con todo tipo de personas, yo creo que sí le sirve harto". (Fuente: Madre Informante Clave 3)

El fútbol en el Club Palestino, comprende un proceso educativo cuyos principales efectos son conocer nuevas personas, amistades, y significar una actividad saludable y de ocio. Sin embargo, existe una distinción importante en la comprensión de convivencia en la madre del informante clave 3 , la que afirma que el fútbol le transmite a su hijo un aprendizaje indirecto y a largo plazo, desde la inclusión del niño en un contexto de fútbol de barrio para conocer a las personas que ahí se encuentran, y observar los modelos negativos de comportamiento que allí se generan para aprender lo que no se debe hacer.

\subsubsection{Dinámicas de relación en el contexto del fútbol de barrio}

Definimos dos subcategorías; la primera, referida a los modelos de comportamiento considerados negativos por los informantes y sus padres; y la segunda, el gusto por el fútbol, aspecto positivo dentro del contexto. 
5.2.2.1. Modelos de comportamientos negativos en el contexto de fútbol de barrio

Padres y niños identifican en el fútbol de barrio diversos comportamientos que influyen en la convivencia de la comunidad que rodea al Club. En nuestros registros describimos constantemente modelos considerados negativos, tanto por los padres y niños, en situaciones tales como: "No había respeto, porque el DT mientras los chicos jugaban [...] estaba compartiendo con los amigos una cervecita po'. Entonces que bien quieren que van a tener los chicos como futbolistas [...] empiezan las insolencias, que pa'cá, que pa'llá, entonces [...] empiezan a discutir los... DT, y empiezan a pelear los cabros chicos po" (Fuente: Madre Informante Clave 1). Además: "[...] son hartas cosas que en las canchas de barrio se ven, porque allá la gente tan tomando o tan pitiando, no les importa que hayan niños más chicos (...) las personas adultas somos un ejemplo para los chicos, hay que irlos a apoyar, no irles a demostrar malas actitudes, pero eso no se ve en los barrios". (Fuente: Madre Informante Clave 3)

Tales ejemplos influyen negativamente la convivencia de los niños, coartando, según los informantes claves, su comportamiento futuro, ya que la costumbre de observar estos acontecimientos, constituyen la esencia del fútbol de barrios y por ende, construye la identidad del niño en este ambiente. En consecuencia, la convivencia se transforma con estos modelos negativos de comportamientos, no obstante para las personas implicadas en dichas acciones aparenta ser un hecho positivo que fomenta el compartir y la unión de la comunidad que participa alentando algún Club de barrio.

\subsubsection{El gusto por el Fútbol}

Un aspecto que potencia la convivencia es el gusto por el fútbol, y la motivación de cada niño por participar en los partidos incrementa la relación entre los jugadores. Esto se evidencia en lo siguiente:

[...] le gusta el fútbol, a donde él tenga que jugar, él es así, porque le gusta el fútbol [...] a Marcelo le gustaba harto el fútbol, y él de los 2 años iba a jugar a la Multicancha, y lo llevaba po'. Nosotros decíamos, éste nació con una pelota en los pies, porque siempre no sé de dónde, le encanta el fútbol, si su pasión es eso. (Fuente: Madre Informante Clave 3.)

[...] Después del Colegio se van para la cancha, y se sacan todas esas malas vibras que traen [...] Cuando llega aquí, se cambia de ropa y abajo es su desahogo, en la cancha [...] ahí lo vienen a buscar los amigos del Club y se van a jugar allá abajo [...] a veces el copete está presente antes que la comida po'. Entonces los chicos salen de sus casas por lo mismo po', para no estar discutiendo con los papás [...] como una salida de escape, se relajan pegándole a la pelota" (Fuente: Madre Informante Clave 1)

Deducimos que a los informantes claves les gusta el fútbol, siendo su principal fuente de distracción, entretenimiento, el impulso para levantarse temprano, colaborar con sus compañeros y las dirigentes del equipo. Entonces, la convivencia es fomentada y beneficiada por la práctica deportiva, pues tanto los jugadores como los padres se relacionan en este ambiente donde se incluye a todo el barrio, desde niños y niñas de 6 años hasta adultos de 50 años. Esta disciplina deportiva y genera convivencia e involucra al niño en un ambiente familiar todos los fines de semana. 


\subsection{Conexión deductivo-inductivo}

En el análisis de los tipos de niveles, se desprende un complemento entendido como convivencia. Se infiere que en el Club Palestino se desarrolla dicho concepto a partir del sentido de pertenencia de los sujetos participantes con la comunidad, fomentada a través de la visión familiar que éstos tienen con respecto al Club: "Así lo veo en barrios, Palestino y nunca me voy a cambiar a otro equipo, porque igual yo vivo en Los Jazmines, así que nunca me cambiaría de barrio" (Fuente: Informante Clave 2), “¿Cómo definirías que son ustedes, como grupo? (Una familia) (Fuente: Informante Clave 1), "nosotros siempre hemos sido de acá de, de Los Jazmines y pertenecemos al Club de por siempre, entonces yo en realidad no, he sacado a mi hijo a jugar a otro equipo" (Fuente: Padre Informante Clave 2).

Para los sujetos el Club es una familia, lo que tiene directa relación con pertenecer al barrio Los Jazmines, ya que esto es el principal argumento del vínculo que sostienen con el equipo, dentro y fuera del ámbito deportivo. Dentro de la convivencia, Jiménez Romero (2005) señala que: "Lo compartido; [...] la pertenencia política o cultural, que nos da una clara conciencia de que, aunque somos diferentes, somos semejantes en otros aspectos, como ciudadanos de un país, miembros de un barrio o escuela [...] que nos dan identidad y pertenencia. Así mismo, se encuentra otro factor que contribuye a desarrollar dicho concepto, el cual corresponde al gran número de mujeres que integran la comunidad del equipo. Esto se manifiesta a nivel administrativo (Presidenta y roles complementarios), y en las mujeres que acompañan a los jugadores cada fin de semana. Lo anterior, se evidencia en "¿Cree que ésta comunidad de Los Jazmines, tiene algo en especial? Sí [...] porque tú ves que las primeras que llegamos abajo, son las mujeres. Tú empezai un partido, y las primeras que están abajo son las mujeres po' (Fuente: Madre Informante Clave 1).

Esto le otorga originalidad con respecto a otros equipos, reforzando el sentido familiar conferida a la comunidad de Los Jazmines y su proyección en el equipo. Además, el fútbol permite complementar los objetivos con que asiste cada niño a jugar, independiente lo diverso que estos sean. Este deporte constituye una herramienta de relaciones y convivencia, uniendo a los niños bajo un mismo ideal en el juego, que, en este caso, es el amor por su institución. En torno a esto, las expectativas de cada uno, se complementan en el ímpetu por ganar cada partido, "defendiendo su camiseta" en cada encuentro. Esto, se ve expuesto en las siguientes referencias:

Informante clave 2: Divertirse con los amigos, si ese es el fútbol al menos [...]

Entrevistadora: ¿tú consideras que en el Club tienes amigos?

Informante clave 2: Sí, tengo hartos, casi todo el equipo..." (Fuente: Informante Clave 2)

Entrevistadora: ¿Entonces tú vas a jugar porque te divierte jugar?...

Informante clave 1: No, es que yo siento garra, siento pasión..." (Fuente: Informante Clave 1)

Entrevistador: ¿para ti, que son tus compañeros de equipo?

Informante clave 3: Como familia así, en la cancha" (Fuente: Informante Clave 3)

Sin embargo, el hecho de constituir una población desfavorecida socialmente determina lo que las personas externas al barrio sostienen sobre la comunidad del equipo. Para ellos no existe convivencia dentro del Club, ya que desde el caso del informante nํㅜ, las dinámicas de 
relación que sostienen las personas de la comunidad de Los Jazmines significan aprendizajes socialmente negativos. Esto se distingue en:

De repente decía, me invitaron, por ejemplo, chiquillos que jugaban con él en Palestino... no, usted ahí en la cancha, y después se olvida de los amigos [...] ¿Cómo tener relaciones de amistad? No, pero de equipo sí. De amistades, de amigos de que... no. No me gustan los chicos para amigos para el Informante clave $3[\ldots]$ porque hay niñitos que no tiene respeto con nadie, no. (Fuente: Madre Informante Clave 3)

¿A quién, por ejemplo, defines tú como tu amigo dentro de Palestino? (Amigo no tengo, es que no tengo la confianza como para decirles algo. Amigos se les llama a los que están al lado tuyo, en las buenas y en las malas, pero ellos son como para diversión (Fuente: Informante Clave 3).

De este modo, quienes residen ajenos a la población no conviven dentro del Club, y más bien, concurren a jugar con el objetivo de cumplir con un compromiso personal 0 un propósito intrapersonal, como el hecho de ganar. A pesar de esto, la convivencia de los niños que son parte del barrio no se ve afectada por este caso excepcional, puesto que demuestran aceptación ante la diversidad de los demás, lo que se manifiesta a nivel intrapersonal e interpersonal, en la vida cotidiana con la comunidad. El Informante 3 a nivel intrapersonal, desde su relación con los jugadores del Club, logra satisfacer su propósito de ganar cada partido, pero a un nivel interpersonal, no se proyecta hacia los demás, es decir no convive con los compañeros de equipo.

A continuación, se expone un mapa axial, diseñado para configurar la conexión de las categorías y subcategorías deductivas e inductivas, correspondientes al análisis y la interpretación de la información donde se articulan globalmente, los conceptos fundamentales del objetivo de investigación, referido a la comprensión de la convivencia que sostienen jugadores del Club Palestino.

Figura 4 - Mapa Axial de categorías.

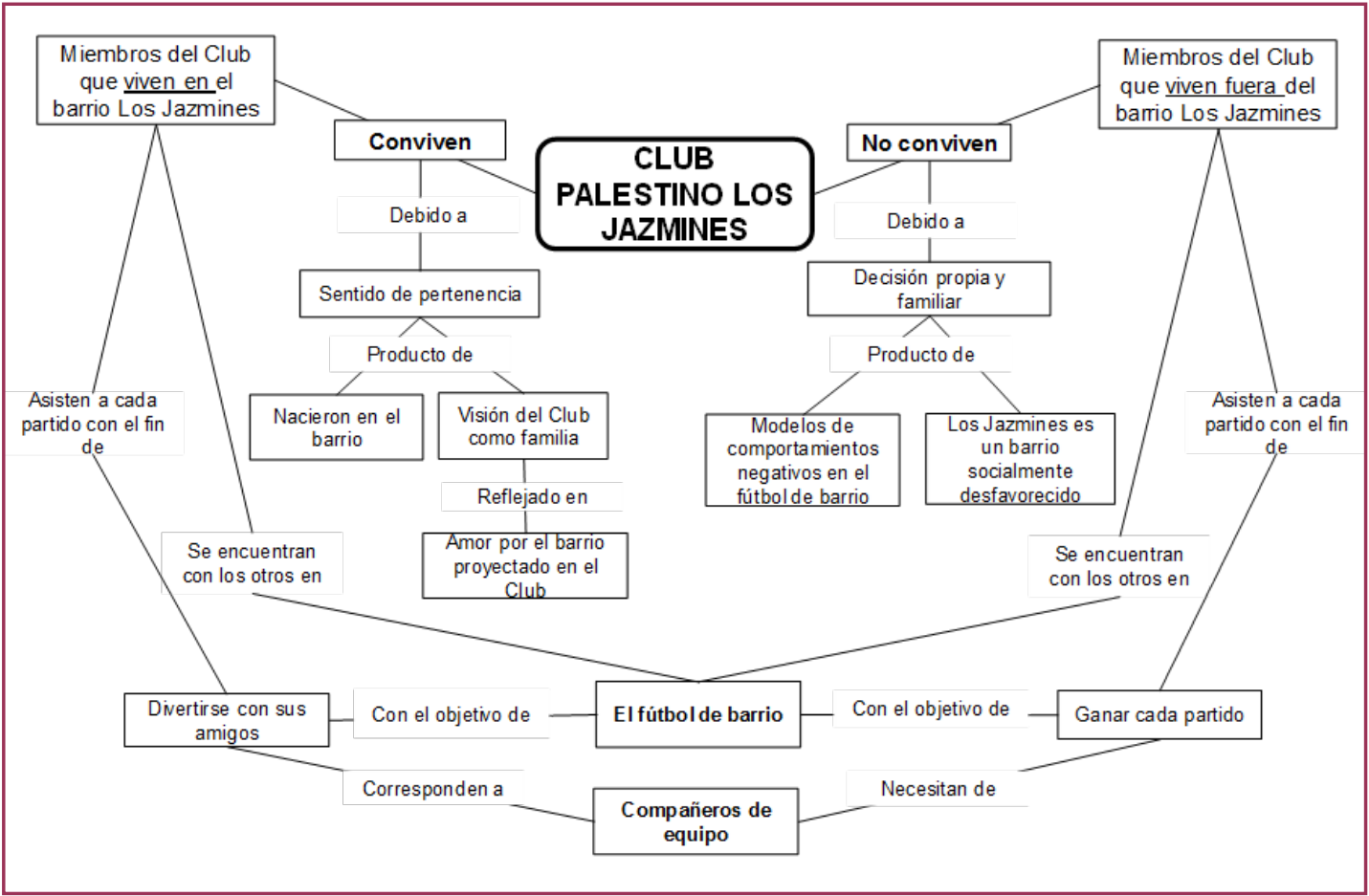

Fuente: Autores 


\section{CONCLUSIONES}

Respecto a las preguntas de investigación, el fútbol constituye un proceso educativo que permite apropiar aprendizajes desde diversos contextos y relaciones, por ejemplo, la adquisición de hábitos como la puntualidad, ocupación del tiempo libre, disciplina, entre otros. Distinguimos dos factores importantes y opuestos entre sí, los cuales dan respuesta a la interrogante respecto a la potencialidad educativa o de vulnerabilidad del fútbol, ya que por un lado, actitudes que generalmente manifiestan los adultos en este contexto, como por ejemplo la ingesta de alcohol y drogas, y manifestaciones violentas o groseras con sus pares o hacia los mismos jugadores, son modelos de comportamientos negativos en los niños, lo cual limita los procesos de convivencia. Por el contrario, el gusto y afición por el fútbol que evidencian los niños, es un factor que genera instancias de interacción social entre los miembros del Club.

En cuanto a los beneficios que obtiene un Club al desempeñarse en él, personas en formación profesional, destacan la dedicación, el compromiso, el respeto, la compañía, el afecto, entre otras. Esto se evidencia en que a partir de la Investigación-Intervención, disminuyeron los insultos y manifestaciones violentas entre los integrantes del equipo. De igual manera, para la formación profesional de los investigadores implica desarrollar responsabilidad, compromiso y lazos afectivos, en tanto que supone una necesidad por parte de los niños que los investigadores persistan como monitores.

En conclusión, se identificaron dos grupos en el Club: los residentes del barrio Los Jazmines y los que viven externos a él. Los primeros conviven con la comunidad del Club, compartiendo un sentido de pertenencia, puesto que al vivir en el barrio le adjudican un sentido familiar al equipo reflejado en el amor que demuestran hacia la comunidad. De esta manera, la diversión que experimentan en el juego con sus compañeros, es relevante para asistir a los partidos, puesto que los procesos de convivencia suceden a partir de las dinámicas de relación social cotidianas, porque además de ser compañeros de equipo, son vecinos, amigos, compañeros de curso, familiares, etc. Por otro lado, quienes viven externos al barrio Los Jazmines, la relación que sostienen en la comunidad no trascienden más allá de lo deportivo, ya que no conviven en el Club y si lo hacen, es en función de mejorar competitivamente y obtener logros deportivos. Esto se evidencia en cada partido donde los que viven fuera del barrio, luego de jugar abandonan el recinto inmediatamente, en cambio, quienes pertenecen a la población, se quedan a compartir con sus compañeros. Sin embargo, ambos grupos se encuentran en el fútbol de barrio, estableciendo relaciones en torno al deporte, permitiéndoles unirse por un objetivo común que es ganar el partido por el Club que representan.

\section{REFERENCIAS}

ALVARADO, Lusmidia; GARCÍA, Margarita. Características más relevantes del paradigma sociocrítico: su aplicación en investigaciones de educación ambiental y de enseñanza de las ciencias realizadas en el Doctorado de Educación del Instituto Pedagógica de Caracas. Sapiens, Revista Universitaria de Investigación, v.9, n.2, p.187-202, 2008.

ALBERICH, Tomás. Investigación-Acción Participativa y Mapas Sociales. Benlloch (Castellón): Ponencia, 2007. 
BALIBREA, Kety; SANTOS, Antonio. El deporte en los barrios ¿integración o control social? Valencia: Editorial Universitat Politécnica de Valencia. 2010.

BUENO, María; LORENZO, Macarena. Entrenamiento de habilidades sociales en el Fútbol Base: Propuesta de Intervención. Revista Internacional de Humanidades y Ciencias Sociales SOCIOTAM, v.21, n.2, p.39-52, 2011.

CORONIL, Almudena. El desarrollo de las habilidades sociales como estrategia para la integración en el grupo-clase en la Educación Secundaria. Ceuta: Curso, 2007-2008.

GIL, Francisco y LEÓN, José María. Habilidades sociales: teoría, investigación e intervención. Madrid: Síntesis. 1998.

GUTIÉRREZ, Belén. Manual de evaluación y entrenamiento de las habilidades sociales para personas con retraso mental. Valladolid: Consejería de Sanidad y Bienestar Social, 2002.

HERNÁNDEZ, Antonio. y TAPIA, A. Fútbol: Concepto e Investigación. Revista Digital EFDeportes. com. Año 15, n.148. Buenos Aires. 2010. Disponible en: <http://www. efdeportes.com/efd148/futbolconcepto-e-investigacion.htm>. Acceso en: 20 ago. 2014.

JIMÉNEZ ROMERO. Convivencia Intercultural de la Cuidad de Madrid, España. Puntos de Vista Cuadernos de Observatorio de las migraciones de la Convivencia intercultural de la Cuidad de Madrid, n.1, p. 7-31, 2005.

MARTÍNEZ, Miguel. Dimensiones Básicas de un desarrollo humano integral. Revista de la Universidad Bolivariana, v.8, n.23, p.129-138, 2009.

MATURANA, Humberto. Transformación en la convivencia. Santiago de Chile: Dolmen, 2000.

PACHECO, Jose Antonio et al.. Habilidades Sociales. In: Plan de acción Tutorial: Gades. Cadiz: Junta de Andaluzia, 2000. p.134-159.

RITTERSTEIN, Pablo. Aprendizaje y Vínculo: Una mirada sobre el aprendizaje: Enrique Pichón y Paulo Freire. Buenos Aires: Universidad de Buenos Aires .Facultad de Ciencias Sociales. 2008.

RUGGIO, Germán. La corporeidad: el nuevo paradigma como formador de la identidad humana. Revista Digital EFDeportes.com., v. 16, n.161, 2011. Disponible en: <http://www.efdeportes.com/ efd161/la-corporeidad-el-nuevo-paradigma.htm>. Acceso en: 10 jul. 2014.

SANTIAGO, Juan. El desarrollo de Valores Sociales positivos dentro del deporte escolar. Revista Digital EFDeportes.com.,v. 8, n.47, 2002. Disponible en: <http://www.efdeportes.com/efd47/valores. htm>. Acceso en: 14 ago. 2014.

SCHUTZ, William. Todos somos uno: la cultura de los encuentros. Buenos Aires: Amorrortu, 2001.

TORO, Sergio. Neurociencias y aprendizaje, un texto en construcción. Revista Estudios Pedagógicos, v. 37, n.2. p.313-331, 2010.

VASILACHIS, Irene. Estrategias de investigación cualitativa. Barcelona: Gedisa, 2006. 


\section{Financiacion:}

Trabajo desarrollado en el contexto del proyecto Fondecyt № 1120761 "Conocimiento y Comunidad Escolar: Procesos de Interpretación de las Dinámicas Relacionales Escolares en la Educación Básica como Configuración Cognitiva Situada" de la Universidad Austral de Chile. 